\title{
Ouvrages en débat
}

\author{
La connaissance de la vie aujourd'hui \\ Jean Gayon, Victor Petit \\ ISTE editions, 2018, 531 p.
}

«Connaître » est le sujet de ce livre. La «biologie» n'est que l'objet de la connaissance même si l'image d'une double hélice d'ADN sur la couverture laisse imaginer autre chose. «Aujourd'hui » annonce un regard sur le présent d'une discipline qui a eu un passé agité. Jean Gayon, premier auteur, est en biologie l'inspirateur d'un courant de pensée, d'une école, qui associe histoire et philosophie ou philosophie et histoire. Cette conjugaison inspire les penseurs de sa génération comme les chercheurs plus jeunes. Victor Petit en est un.

Ce livre examine l'acte de connaître et sa dépendance vis-à-vis des acquisitions passées et vis-à-vis des échanges réciproques entre la science qui se fait, et la société qui évolue. V. Petit en a eu l'initiative et lui a donné la forme d'entretiens où il interroge J. Gayon. À l'époque où l'exercice a débuté, les auteurs ignoraient que l'ouvrage serait en 2019 un testament scientifique. De fait dans les dernières pages, sachant que son temps était compté, J. Gayon a fermement défendu ses convictions d'historien et de philosophe, son appréhension de la biologie et sa vision des affaires du monde.

V. Petit a conduit les entretiens par ses questions, puis conçu la structure du livre. J. Gayon en a approuvé le projet. L'histoire de la carrière et des travaux de J. Gayon est le fil conducteur. Présenté en six chapitres («Études, parcours», "Histoire et philosophie des sciences», "Philosophie de la biologie», "Autour de Darwin", «Sur la génétique», «Biologie et société»), ce choix oblige à quelques redondances. Parfois la nuance des redondances éclaire les questionnements avec plus d'acuité, sur la place de l'aléatoire dans l'évolution, par exemple.

Bien identifiées par une typographie bleue, les questions de V.Petit constituent en elles-mêmes un sous-ensemble. Celui-ci témoigne d'un exercice difficile, mais il oriente l'ensemble du livre. Pour J. Gayon les faits sont là et leur connaissance est le fruit d'une histoire. L'analyse scientifique de cette histoire est indispensable à l'exégèse philosophique. Cette dernière ouvre le champ de conjectures et initie questionnements et actes de connaître nouveaux. Le balancement, ses limites et sa fragilité se perçoivent dans la succession choisie des questions et des réponses. Le balancier penche vers l'histoire dans le chapitre sur l'évolution, vers l'exégèse dans celui traitant de société et santé, par exemple.

Dans le chapitre «Histoire et philosophie des sciences », V. Petit demande comment s'est réalisée la conjugaison des deux disciplines. J. Gayon raconte que le «je pense que...» a rencontré les faits avant d'en proposer des représentations. On peut en rester là, mais il est aussi possible de poser plus de questions, dans un paysage où s'articulent rationnel et irrationnel, sur les rôles respectifs du milieu et du hasard, dans l'évolution ou la génétique, par exemple. J. Gayon assortit ce propos d'une mise en garde appelée bornage : les frontières entre les disciplines sont perméables mais fluctuantes. Dès lors, comment choisir sa démarche (universalité ou parcellisation disciplinaire) ? Comment évaluer le poids spécifique du passé, celui des idées présentes et des pratiques installées, celui de l'intrusion de techniques venues d'ailleurs? En matière d'objets biologiques J. Gayon propose de mener l'analyse et la reconstruction historique en association avec la formulation d'une raison constituante. Gène et sélection serviront à l'exercice.

Le chapitre «Philosophie de la biologie» affirme que l'histoire est un bon vecteur d'échanges entre philosophie et biologie, vecteur où le temps joue un rôle majeur dans leur conjugaison. La leçon serait que les systèmes philosophiques peuvent jouer le rôle de fonctions à qui l'on demande à la fois des représentations de faits biologiques et des prédictions sur leur évolution. À la formalisation mathématique d'un fonctionnement probabiliste et stochastique du vivant les systèmes philosophiques apportent une régulation contingente. Elle permet d'écarter des évolutions impossibles, des évolutions que nous n'anticipons pas. Sous cet angle sont discutées les «lois de Mendel» : elles se présentent comme des lois empiriques et circonstancielles et ne sont pas des lois au sens propre de la philosophie des sciences. Et les interrogations de se succéder: le principe de sélection naturelle est-il une généralisation empirique ou une application à des situations empiriques ? Définir la vie a-t-il un sens ou faut-il construire des systèmes à partir d'organismes vivants ? Il apparaît que «la vie» comme «l'information biologique» sont des 
commodités pour satisfaire un besoin d'universalité et de mise à l'épreuve. Ces propos éclairent l'intérêt de la formalisation mathématique et de la modélisation, par exemple en génétique des populations ou pour l'usage de données massives.

Vient ensuite l'œuvre majeure de J.Gayon: deux chapitres, «Autour de Darwin », et "Sur la génétique». Sur ces sujets J.Gayon a acquis une réputation internationale, une reconnaissance de maître à penser. Présenter les deux sujets dans cet ordre est justifié parce que le concept de sélection naturelle a précédé celui du gène actuel (gène porté par son $\mathrm{ADN}$ ). Pourtant ce sont la génétique des populations, la génétique moléculaire et les avancées techniques qui ont sorti les études sur l'évolution du simple constat et de la classification, et leur ont donné une dimension prospective.

«Autour de Darwin» est une pièce en deux actes. Premier acte: l'analyse historique sur l'œuvre et la pensée, sur les relations positives et négatives avec son temps. Le principe de sélection naturelle est une immense nouveauté. Son mérite est de ne pas être dépendant d'un objet biologique particulier. Il offre des réponses provisoires aux questions des biologistes et des philosophes. Il éclaire le phénomène établi de l'adaptation. Acte second et nouveau saut: une formalisation mathématique universelle est devenue possible et le gène est l'unité sur laquelle elle porte. La génétique des populations va éclairer, scientifiquement, à la fois les processus évolutifs et les processus écologiques. Envisagée au niveau des gènes au début, la sélection s'adresse tout autant aux entités écologiques, aux espèces qu'aux cellules, aux organismes et aux acteurs moléculaires. Comment savoir ce qu'est la sélection? À la recherche de faits, les uns vont se plonger dans l'analyse circonstancielle. Les autres vont développer le concept de valeur sélective (fitness) pour s'intéresser directement aux niveaux de complexité les plus élevés. J. Gayon traite magnifiquement de cette aventure. En choisissant ses questions, en leur donnant une formulation rhétorique, il arrive parfois que V. Petit écarte l'attention du lecteur de l'objectif principal (cas de la sélection sexuelle, par exemple).

«Sur la génétique » est aussi une pièce en deux actes. L'histoire du gène et la quête de son identité (identité attendue puis perdue) en est le premier. L'histoire de la génétique en France est le second. Sur ces deux sujets J. Gayon a éclairé les connaissances biologiques. Sur ces deux sujets biologistes et médecins cherchent des références dans ses travaux (même si J. Gayon n'a pas traité directement de santé et de médecine). Mendel a découvert le gène sans le nommer. Au fil des années, le gène deviendra une réalité formelle pour les généticiens des populations, chromosomique pour les généticiens «fondamentaux», moléculaire pour les biologistes du même nom. Ces définitions successives et délicatement différentes sont l'objet d'une recherche tripartite biologiehistoire-philosophie. Les technologies (par le séquençage de l'ADN particulièrement) ont bousculé les faits et les idées. Aujourd'hui, le gène apparaît comme un concept syncrétique dont l'ADN est un élément matériel et l'information un élément formel bien que concret. Ces développements reposent la question : que faut-il entendre aujourd'hui par «déterminisme génétique»? J. Gayon revient alors sur le sens de ces deux mots. L'usage en biologie en a dérivé par rapport au sens général donné en philosophie des sciences, mais l'utilité de la formule est incontestable. Sur cette base J. Gayon met systématiquement face à face génétique des populations et génétique physiologique (ou moléculaire). Aujourd'hui le temps paraît venu de concevoir un futur par le développement simultané et coordonné des trois disciplines (histoire, philosophie, biologie) et de traiter des possibilités de déconstruire le gène tout en conservant le concept pour aborder en termes nouveaux la question de la «transmission héréditaire». La première partie est à la mode, épigénétique aidant (celle-ci était d'ailleurs connue avant l'ADN et portait alors souvent le joli nom de «pénétrance »). Conserver le concept revisité ouvre d'autres perspectives : reprendre l'idée d'adaptation en sortant du vieux conflit qui la charge, transformer les données massives du séquençage en faits biologiques, ouvrir le champ de la dynamique des entités écologiques. Ces réflexions poussent le lecteur à regarder encore une fois l'image de la page de garde : y sont présentées une double hélice et une cascade de nucléotides alors que ce ne sont pas les sujets du livre, mais on distingue aussi un trait sombre qui borde la page de nucléotides et ondule avec la double hélice. Il pourrait s'agir d'un clin d'œil à « la triple hélice ${ }^{1}$ » de Richard Lewontin et/ou à l'implication du milieu dans le déterminisme génétique...

Le livre se ferme sur un chapitre intitulé « Biologie et société ». L'exposé change alors de style. V. Petit insiste pour que J. Gayon prenne position sur des questions très actuelles (toutes ne sont pas biologiques): hérédité, environnement et idéologie, eugénisme et transhumanisme, évolution humaine, biotechnologies. Le propos est moins affirmé. L'historien et le philosophe ont un problème difficile: l'analyse et l'exégèse classiques peuvent-elles suivre le rythme d'arrivée des connaissances poussée par les techniques? Un exemple peut illustrer le propos: en 2017 quand V.Petit interrogeait J.Gayon, on savait déjà que la synthèse de bases organiques autres que $\mathrm{A}, \mathrm{T}, \mathrm{C}$ ou $\mathrm{G}$ était possible,

\footnotetext{
${ }^{1}$ Lewontin R.C., 2003. La triple hélice. Les gènes, l'organisme, l'environnement, Paris, éditions du Seuil. Traduit de: The triple helix. Gene, organism, and environment, Cambridge (MA)/London, Harvard University Press.
} 
qu'elles pouvaient s'insérer dans un ADN et installer un acide aminé original dans une protéine. Ce n'est pas évoqué. Pourtant, sur le plan "philosophique» cela suggère que d'autres "codes» que le nôtre sont possibles. La sélection et le hasard n'en auraient-ils retenu qu'un, celui des organismes actuels? Historiens et philosophes n'ont pas fini de se pencher sur la biologie de synthèse, sur l'évolution et sur les libertés qu'elles promettent... À ce constat, J. Gayon répond en ouvrant de nouveaux chantiers théoriques (la réversibilité, par exemple), en exigeant qu'en s'inspirant de la science historique, les propositions formelles nouvelles aient une capacité prédictive.

Jean-Claude Mounolou (Directeur de recherche honoraire, CNRS, CGM, Gif-sur-Yvette, France) mounoloujcm@orange.fr

La part sauvage du monde. Penser la nature dans l'Anthropocène

Virginie Maris

Éditions du Seuil, 2018, 259 p.

Ce livre, rédigé par Virginie Maris, philosophe de l'environnement, est un véritable plaidoyer pour la part sauvage du monde, à rebours des conceptions modernes de la fin de la nature et de l'omniprésence de l'artifice. Il peut se lire aussi comme une approche critique du discours sur l'Anthropocène qui véhiculerait une vision du monde en conformité avec l'idéologie néolibérale qui le gouverne. Afin de nous convaincre de la nécessité de conserver et de respecter cette part du monde que l'homme n'a pas créée et qui demeure étrangère à ses finalités, l'auteure nous propose de l'examiner dans sa plus grande altérité, en lui réattribuant le terme de nature qui a été délaissé au profit de concepts comme celui de biodiversité puis de services écosystémiques. Le défi intellectuel que se donne V. Maris est de retrouver le " chemin de la séparation dans un monde hyperconnecté », ce qui exige une attitude réflexive sur la nature que nous protégeons et la manière dont nous la concevons. Alors que nous pensions nous être enfin débarrassés de ce dualisme nature/culture, voilà que l'auteure nous met en garde sur les biais et les limites de cette position de retrouvaille pour nous convaincre de nous aventurer sur ce chemin étroit, entre réalisme naïf et naturalisme moral, où la défense du sauvage est associée à un projet d'ouverture à l'autre, de critique du capitalisme et d'émancipation.

Dès l'introduction, on saisit la difficulté d'une telle approche qui suppose une distanciation de l'auteure avec ses alliés habituels, ceux qui s'occupent du vivre ensemble, de la défense des paysanneries et des populations indigènes. Elle s'emploie à nous rassurer en précisant qu'il n'est pas question de disqualifier ces chantiers indispensables, mais de montrer qu'ils doivent cohabiter avec cet horizon sauvage dont aurait besoin l'humanité pour savoir mieux habiter la Terre. La thèse défendue peut se résumer à cette question : comment interagir avec la nature et borner en même temps l'empire humain ? Le parti pris est donc de revisiter le grand partage, rendu responsable de la crise occidentale, puis d'analyser des éléments de discours sur la fin de la nature pour constater, ou non, la fin de toute extériorité à l'agir humain. La grande originalité de ce livre est de reprendre l'histoire de la conservation selon une posture « renversée », en observant les processus d'absorption de la nature et sa progressive dissolution dans les sphères à la fois techniques, économiques et informationnelles. Pour tenter de clarifier ce débat complexe, l'ouvrage est organisé en quatre grandes parties, elles-mêmes divisées en sous-chapitres. Chacune s'empare d'une question en particulier et s'emploie, références bibliographiques à l'appui, à la documenter et à en faire l'analyse. L'auteure mobilise l'histoire des idées, la philosophie, la science et ses avancées pour affûter ses arguments et effacer tout malentendu quant à l'interprétation de son objet.

La première partie, intitulée «Le grand partage», retrace à grands traits le concept de nature et son évolution à travers le temps dans ses dimensions philosophique, scientifique et politique et insiste sur les différents sens qu'il a pris dans l'histoire de la pensée. Quand la nature se confond avec la part du monde que nous n'avons pas créée, elle est qualifiée par l'auteure de «nature-altérité». L'idée centrale est de se faire autant critique que l'avocat du dualisme occidental parce qu'il repose justement sur la reconnaissance d'une altérité radicale du monde naturel qu'il est difficile de nier en bloc. Ainsi, considérer l'homme comme un perturbateur des écosystèmes sauvages, d'une part, et reconnaître son pouvoir de destruction sur l'ensemble du monde sauvage, d'autre part, s'inscrit dans cette dualité. Il faudrait aussi ne pas oublier la relative centralité de l'humanité sur la grande scène biologique. Au XIX siècle, sous l'influence des travaux de Darwin, trois thèmes principaux ont un impact considérable sur l'idée de nature-altérité : l'historicité de la nature, la continuité de l'humain avec les autres êtres vivants et l'interdépendance entre les vivants et leur milieu. Si les premiers défenseurs de la nature se font entendre dès cette époque et restent longtemps tributaires d'un certain dualisme, les approches plus récentes de la conservation de la biodiversité vont s'affranchir de cette séparation entre les humains et la nature. Bref, plus qu'à un grand partage entre nature et culture souvent invoqué pour justifier les crises environnementales, la lecture de cette partie nous confronte en réalité à un "grand bazar» que l'auteure va tenter d'explorer de façon synthétique mais dans toute sa complexité. 
La deuxième partie, «La fin de la nature », revient sur les bases conceptuelles et empiriques de ce grand partage qui en soulignent les limites et dont la fin de la nature serait l'expression. La relecture rapide des apports de Latour et de Descola quant à la multitude des enchevêtrements entre entités de nature et finalités humaines n'empêche pas V. Maris de plaider pour le maintien d'une nature-altérité comme concept référent. Néanmoins, le véritable danger de son effacement viendrait essentiellement des sciences de l'Anthropocène qui, en posant le principe d'une pensée systémique à l'échelle de la planète ou de l'espèce, justifient des solutions techniques et scientifiques uniformes à la crise environnementale. Si la forte intrication des processus naturels et des processus culturels a fait l'objet de multiples travaux dans le passé, comment penser le statut des sciences qui prétendent rendre compte de ces influences mutuelles à l'époque de l'Anthropocène? Comme nous le rappelle l'auteure, les voies sont multiples et risquées. Retenons l'émergence d'une « anthroécologie » qui observerait sous un nouvel angle la biosphère « systèmes humains incorporant des écosystèmes naturels en leur sein ». Se dessine un ensemble de prescriptions sur ce qu'il conviendrait de faire pour ne pas mettre en péril l'humanité. Les penseurs de l'Anthropocène peuvent nous inquiéter puisqu'il s'agit ni plus ni moins d'admettre la fin de la nature et de la culture. Poursuivant sa réflexion, l'auteure interroge le devenir de l'écologie et de la conservation face à ce nouveau monde. Là encore, et pour enlever toute ambiguité, elle valide les approches reposant sur les socioécosytèmes dans la mesure où ils représentent une grande partie du monde dans lequel nous vivons, mais elle plaide pour l'étude des systèmes peu ou pas influencés. De façon paradoxale, parce que soucieux d'une nature qui soit compatible avec les attentes des hommes et favorise leur propre développement, le mouvement de la «nouvelle conservation», en se ralliant au projet de domestication complète du monde, contribuerait à la disparition de la nature par un phénomène de triple «absorption» (objet de la troisième partie) dans les sphères de l'agir humain.

La troisième partie, organisée en trois chapitres, traite successivement de ces phénomènes d'absorption par les entrées technique, économique, et le traitement scientifique. Ces trois mutations ont transformé la nature en artefact, en marchandise et en banque de données au sein même des institutions et des communautés scientifiques et professionnelles qui avaient placé la protection de la nature au fondement de leur mission. À travers le filtre du progrès des techniques, le premier chapitre revient sur les différents outils de la conservation et l'évolution des politiques d'aires protégées. C'est l'occasion pour V. Maris de reprendre le binôme naturel/artificiel et de faire l'éloge des apports de la notion de continuum qui a permis de se préoccuper de la nature ordinaire, de multiplier les approches en termes de services écosystémiques ou encore de développer l'écologie urbaine. Mais là encore, le risque est grand de marginaliser les plaidoyers en faveur d'une nature plus sauvage et moins accessible. C'est l'éventail même des possibles qui s'en trouverait réduit. Les nombreux débats et conflits de valeur recensés dans l'ouvrage autour de l'usage de ces outils portent également sur la distinction entre influences indirectes des activités humaines et transformation intentionnelle que l'auteure considère comme une ligne de partage utile pour penser la conservation et les moyens qui lui sont attribués. Si elle reconnaît l'importance de maintenir en bon état les milieux dans lesquels nous vivons, elle insiste sur la nécessité de maintenir des entités et des territoires à l'écart des productions intentionnelles et indépendamment des bénéfices que l'on peut en tirer. $\mathrm{Ne}$ pas chercher à fabriquer de la nature coûte que coûte, mais l'accompagner dans ses méandres. Les deux chapitres suivants ( $« \mathrm{La}$ nature comme marchandise » et « La nature comme base de données ») sont construits sur le même schéma. Notons que nous sommes au début des recherches sur la mise en données du monde naturel associée à la massification de l'information, autrement dit l'ère du big data. Les innovations technologiques comme l'enrôlement des citoyens amateurs de nature dans la constitution des grands inventaires de biodiversité ont démultiplié la taille des bases de données sur la biodiversité. Reprenant l'histoire naturelle à travers la constitution des collections par les naturalistes, V. Maris interroge les modes de production des connaissances et les objets de la connaissance eux-mêmes : numérisation, standardisation et décontextualisation de la donnée. Outre une perte importante d'informations, un autre risque est de rendre invisibles certaines données réticentes au classement. En fin de parcours, les bases de données créent en partie le monde qu'elles prétendent observer et construisent un nouvel objet scientifique, la biodiversité globale. Les dispositifs institutionnels qui accompagnent ces évolutions permettent le déploiement d'un projet de surveillance globale du système Terre - un biopanoptique géant - qui est encore une façon de nier la nature. La réconciliation avec la nature, très en vogue aujourd'hui, signifie-t-elle tout contrôler? Là aussi, il ne s'agit pas de s'opposer à la promotion de façons de vivre bénéfiques pour les sociétés humaines et pour les autres espèces, mais d'envisager les limites de notre emprise et de conserver une référence à la nature sauvage pour ne pas céder au «syndrome de la référence glissante », proche de ce que Martha Nussbaum décrit comme des préférences adaptatives à l'œuvre dans notre conception de ce qu'est le bon état de la nature.

Dans la quatrième partie, «La part sauvage», V. Maris revient sur les trois attributs de la nature-altérité, à savoir son extériorité, son altérité et son autonomie, pour ensuite définir ce que son projet n'est pas. Reprenant les travaux de Val Plumwood, philosophe et militante écoféministe australienne, sur le dualisme, elle propose 
de penser la nature dans un « régime de différenciation non hiérarchique » qui permette de distinguer la part sauvage du monde. Le rôle des frontières en contrepoint d'un monde globalisé est repensé, la multiplicité des mondes et des points de vue est mise en avant et l'idée de souveraineté du monde sauvage est suggérée. Devançant les nombreuses critiques dont elle risque d'être la cible, comme le sont les politiques préservationnistes, l'auteure prend soin de recadrer son analyse en se déclarant hors du champ de la pensée fixiste, misanthrope et néocoloniale.

Tout au long de cet ouvrage, l'auteure plaide pour cette nature sauvage tout en sachant ce projet périlleux, parce qu'il risque d'être mal compris, voire relégué au camp de l'écologie radicale. Malgré les apories dans lesquelles elle nous plonge, il nous semble que la posture défendue par V.Maris, qui navigue au gré d'un mouvement de balancier entre dualisme de la modernité et socioécosytèmes, est plus subtile que cela, même si conceptuellement elle peut heurter ceux qui s'inscrivent dans l'interdisciplinarité ou le courant des humanités environnementales. L'entrée dans l'Anthropocène légitime les rapprochements entre écologues, économistes, sciences humaines et sociales et on ne peut que s'en réjouir tant que le projet se tient à distance d'une instrumentalisation de la nature, dans la continuité, en fin de compte, de la conception moderne.

Florence Pinton (Université Paris Saclay, UMR1048 SADAPT, Paris, France) florence.pinton@agroparistech.fr

\section{Le mal du dehors. L'influence de l'environnement sur la santé} Rémy Slama

Quæ, 2017, 376 p.

Dans cet ouvrage au sous-titre (L'influence de l'environnement sur la santé) plus explicite que le titre (Le mal du dehors, nous y reviendrons), Rémy Slama (Inserm) relève avec éloquence un triple défi : celui de la complexité, de la temporalité et de l'invisibilité. Complexité, car il est maintenant considéré que la majeure partie du fardeau de la maladie pesant aujourd'hui sur nos sociétés relève d'interactions entre le capital génétique, le comportement et l'environnement d'un individu (mécanismes qu'on commence à peine à comprendre malgré la mobilisation de nombreuses disciplines scientifiques). Temporalité, car le temps historique (l'avènement de l'Anthropocène), le temps d'impact (ou délai de latence, représentant le décalage entre l'exposition à un polluant et la manifestation biologique ou clinique, se mesurant en années, décennies, voire générations - les «effets persistants de substances non persistantes ») [p. 344], et le temps biologique (pouvant être altéré par les interactions précédemment citées) s'entrechoquent. Invisibilité, car pendant longtemps la contamination environnementale ( «les visiteurs silencieux») [p. 351] et/ou les conséquences sanitaires (sans parler de leurs liens) étaient ignorées, faute d'outils adaptés permettant d' $d$ identifier les causes des causes » (p.5). Ce défi est très bien résumé par l'auteur sous la forme de l'«histoire de l'émergence de phénomènes initialement invisibles que la science a su rendre visibles » (p. 13).

Le mal $d u$ dehors est un livre dense (376 pages), assez technique, mais toujours pédagogique, embrassant le continuum recherche, évaluation du risque et gestion du risque. Dans cette entreprise quasi encyclopédique, l'auteur puise dans sa riche expérience de chercheur mais se repose parfois, inévitablement, sur des connaissances plus livresques et donc moins incarnées. Parmi toutes les approches possibles de cette complexité (aussi nombreuses que les combinaisons polluant/exposition/population/pathologie), l'auteur choisit, non sans raison, de doter d'abord le lecteur des concepts-clés et des méthodes cardinales dans le champ de la santé environnementale, pour ensuite aborder les sources, voies d'exposition et mécanismes d'action des contaminants environnementaux. Les effets sanitaires viennent ensuite illustrer toutes ces situations.

R. Slama convoque les grandes figures abondamment citées dans les ouvrages de santé environnementale: le pionnier Percivall Pott reliant en 1775 le cancer du scrotum chez les jeunes ramoneurs à l'exposition aux goudrons des cheminées ; l'incontournable John Snow et la pompe à eau de Broad Street à l'origine de l'épidémie de choléra survenue en 1854 à Londres; ou bien, pour les Français, le précurseur (qu'on qualifierait aujourd'hui de «lanceur d'alerte») Denis Auribault signalant dès 1906 le rôle cancérigène de l'amiante. Mais à chaque fois, R. Slama va un pas plus loin (voire un pont plus loin pour faire écho à l'expression anglaise «to bridge the gap »), apportant le détail inédit qui fait sens et connaissance.

Puis l'auteur passe en revue, de façon systématique et progressive, les différentes disciplines et techniques impliquées (sciences de l'environnement, toxicologie, épidémiologie), pavant une rampe d'accès à la difficile question «Peut-on établir la causalité en santé environnementale ?» (chapitre 8). Ce chapitre se mérite mais le jeu en vaut la chandelle. On retiendra en particulier la métaphore du distributeur de bonbons pour expliquer la relation causale entre polluant et maladie. D'un distributeur habituel (une pièce de monnaie, un bonbon) signant la causalité à l'échelle individuelle à un distributeur pas si inhabituel (une pièce de monnaie, parfois sans bonbon) pour expliquer la causalité à l'échelle de la population (ou «stochastique»), pour aboutir à un distributeur d'un troisième type (qui non seulement ne distribuerait pas 
toujours de bonbons quand on introduit une pièce, mais qui parfois en distribuerait sans l'insertion d'une pièce) pour introduire la notion de causes suffisantes composées (chaque cause prise isolément pouvant être ni nécessaire, ni suffisante). Au cours de ces développements sur la causalité, probablement l'acmé de l'ouvrage, R. Slama sait aussi se faire quelque peu iconoclaste. Il remet par exemple en cause la popularité des neuf critères de causalité décrits par Bradford Hill en 1965, donnant « à certains l'illusion de pouvoir juger de la qualité ou de la pertinence d'une étude épidémiologique [...] sans avoir à entrer dans toutes les subtilités et concepts de la discipline» (p. 136-137).

La partie III du livre concerne les principaux mécanismes d'actions des contaminants environnementaux. Certains aspects fondamentaux pourraient rebuter le lecteur non-scientifique (voire non-biologiste), pourtant les développements sur la régulation de l'expression génétique par des phénomènes épigénétiques valent le détour. Ces caractéristiques épigénétiques sont très sensibles à l'environnement des cellules, des organes et de l'organisme. Des contaminants environnementaux sont ainsi susceptibles d'entrainer des altérations épigénétiques dont le rôle dans la survenue de pathologies commence à être bien documenté.

Les parties IV et $\mathrm{V}$ détaillent les effets des contaminants environnementaux sur la santé. Bien documentées, écrites d'un style alerte, elles se lisent comme la chronique de l'ère industrielle et de sa révolution chimique, de ses espoirs à ses conséquences indésirées.

Dans la dernière partie du livre, l'auteur interroge l'articulation entre science et politique, non sans un certain courage. Il décrypte l'absence d'action publique devant une incertitude scientifique, qui «n'est pas une absence de décision, c'est la décision de ne pas réagir» (p.327). Il montre comment le principe de précaution peut être vu comme la conséquence du choix de ne pas vouloir d'une science forte et ainsi déplacer « le centre de gravité du lieu de prise de la décision vers le politique » (p. 340). Il décrit aussi l'«hétérogénéité manifeste dans les niveaux de risque tolérés en fonction des sources de danger, des secteurs d'activité ou des populations concernées » [p. 317] (il en est ainsi des risques liés à l'exposition de la population générale aux particules fines, pour des raisons non explicitées par les pouvoirs publics). Il rappelle enfin qu'en France, il existe une propension à attendre des citoyens «qu'ils modifient leurs comportements pour limiter leurs expositions plutôt qu'à agir sur l'environnement » (p. 320), prenant exemple sur les ouvriers utilisant une peinture à la céruse (à base de carbonate de plomb).

À l'heure de conclure, R. Slama ne résiste pas à la vision obligée, certes sous la forme d'une pirouette utopiste, d'un «futur optimiste» (p. 354). En décalage avec le pessimisme raisonné qui émane du contenu de l'ouvrage, ce message final apparaît un peu troublé.
Une fois le livre refermé, on peut exprimer deux légers regrets. Si l'auteur embrasse de nombreuses disciplines, et ce n'est pas le moindre de ses mérites, il aborde à peine le rivage des sciences sociales. Il ne consacre qu'un peu plus d'une page aux inégalités sociales de santé et à l'injustice environnementale; la perception du risque (dont l'influence est déterminante dans les comportements vis-à-vis de l'exposition) est juste citée; et nulle mention n'est faite de l'implication des parties prenantes (les «stakeholders» des anglosaxons) dans l'évaluation et la gestion du risque. Par ailleurs, d'où vient ce titre, Le mal du dehors, dont on n'est pas sûr qu'il résulte du choix de l'auteur? À quel «mal du dedans » fait-il miroir? À quel principe moral ou obligation, ce «mal» s'oppose-t-il ? Ou alors serionsnous cernés par les menaces (qu'on traduirait par des «maux»?) franchissant les portes de nos maisons (ou nos barrières biologiques)? Rien ne vient éclairer le lecteur sur ce titre aux acceptions si éloignées. Mais ces quelques réserves ne sauraient ternir l'image de cet ouvrage, unique dans sa profondeur scientifique et historique.

\section{Jean-François Viel (Université de Rennes 1, Faculté de médecine, UMR1085 Irset-Inserm, Rennes, France) jean-francois.viel@univ-rennes1.fr}

\section{The political economy of sustainability}

Fred P. Gale

Edward Elgar, 2018, 284 p.

Fred P. Gale est professeur en sciences politiques et relations internationales à l'Université de Tasmanie. The political economy of sustainability représente l'aboutissement de toute une carrière de réflexion sur les problématiques environnementales, à la croisée de l'économie et de la science politique. L'objectif premier de l'ouvrage est de reconceptualiser la nature de l'économie politique à partir d'une perspective de soutenabilité et ainsi d'œuvrer à une « économie politique de la soutenabilité». Précisons d'emblée qu'il s'agit de fournir non seulement une grille d'analyse des problèmes contemporains à l'usage des chercheurs et experts, mais aussi un socle pour une démarche politique.

La thèse de l'ouvrage est exprimée clairement dans la conclusion (p. 221): «Du point de vue d'une économie politique de la soutenabilité, toutes les activités impliquées dans des chaînes d'extraction-mise au rebut doivent être gouvernées pour assurer l'équilibre de la valeur d'échange, de la valeur d'usage, de la valeur travail et de la valeur fonctionnelle ${ }^{2} »$. L'auteur indexe ces formes de la valeur à

\footnotetext{
$\overline{{ }^{2} \text { Nous traduisons (ici comme par la suite). }}$
} 
des grandes traditions de l'économie politique et de l'écologie auxquelles il reproche un monisme axiologique et une vision tronquée du réel. Il s'agit donc de dépasser les approches traditionnelles de l'économie politique pour faire advenir une «tétraévaluation» («tetravaluation»). Mais avant d'aborder le contenu de cette proposition, il faut comprendre chacune des composantes qui la soustendent, à savoir les formes alternatives de ce que F. Gale appelle la «valeur économique».

L'auteur distingue quatre conceptions de la valeur économique. La valeur d'échange est celle que mettent en avant les économistes libéraux et les néoclassiques, et dont la logique du marché est la juge de paix. La valeur d'usage est quant à elle rapportée à l'idée de puissance productive nationale, et Friedrich List en serait l'un des premiers thuriféraires. La valeur travail, ensuite, qui trouve son origine chez Adam Smith et David Ricardo et son point culminant chez Karl Marx, traduit la double idée que la valeur des marchandises dépend du travail qu'elles ont requis et que les travailleurs doivent obtenir leur juste part des profits contre l'exploitation capitaliste. La valeur fonctionnelle («function value»), enfin, est celle d'une chose (un bien, un service, un acte de production ou de consommation ou encore une politique publique) qui participe au «maintien du système plus large auquel elle appartient»(p.220), qui en d'autres termes contribue aux équilibres écologiques. F. Gale attribue cette valeur à la pensée écologiste de manière générale, dont il propose un tour d'horizon dans son chapitre 3.

Ainsi les innombrables débats sur la valeur et sur les sources de la richesse qui ont animé et animent encore l'économie politique et l'écologie sont-ils synthétisés dans quatre grandes visions du monde, quatre grandes formes de la «valeur économique». Il peut en première approximation paraître étrange de subsumer la pensée écologiste sous une forme de "valeur économique», la valeur fonctionnelle. Il peut aussi surprendre que la valeur d'usage soit rapportée au nationalisme économique et à la puissance de l'État. Mais l'effort héroïque consistant à résumer une telle diversité de théories et de visions du monde dans une tétralogie implique certainement de faire des choix discutables, nous y reviendrons.

Venons-en désormais à ce qui fait l'apport essentiel du livre, la «valeur de soutenabilité $e^{3}$ » ou «valeur soutenable» («sustainability value »). L'idée-phare est que cette valeur hautement désirable se loge à la confluence des quatre formes de valeur exposées

\footnotetext{
${ }^{3}$ Nous préférerons la traduction «valeur de soutenabilité » pour éviter la confusion consistant à penser que la valeur ellemême serait soutenable plutôt que les choses, les pensées ou les actes qu'elle recouvre.
}

précédemment et que son avènement est le fruit de la délibération, de la négociation et du compromis. Les théoriciens qui défendent l'une ou l'autre de ces conceptions de la valeur à l'exception des autres se fourvoieraient, la réalité serait en effet faite de situations où les acteurs entrent dans une "négociation pragmatique trans-valeurs » (p. 220). Donc, en un sens, la valeur de soutenabilité serait déjà mise en œuvre de manière éparse dans le réel, de même qu'elle serait un idéal à poursuivre. Cette assertion à la fois empirique et normative est supposée tordre le cou à toutes les visions partielles et partiales de la réalité économique, coupables d'un biais théorico-idéologique. La «valeur de soutenabilité » serait donc à l'intersection, ou plutôt dans l'équilibre entre ces quatre types de valeurs. F. Gale justifie cette approche par référence au développement soutenable et à ses piliers (chapitre 4), et illustre la diversité des dimensions de ce dernier, qu'il nomme «valeurs», à travers la diversité des indicateurs de soutenabilité existants. Ceux-ci seraient pour la plupart monodimensionnels, ce qui pose problème dans une perspective de tétraévaluation. Or, faire advenir cette dernière suppose de prendre en compte une diversité d'indicateurs, mais également de négocier des arbitrages et d'arriver à travers la délibération et la négociation pragmatique à des «compromis du plus petit dénominateur commun acceptable par tous » (p. 221). Il faudrait pour cela mettre en place des structures adéquates de tétraévaluation à la fois réflexives et délibératives. Et cela, pour gouverner aussi bien l'entreprise (chapitre 6) que la politique (chapitre 7) et le citoyen-consommateur (chapitre 8).

On l'a compris, il s'agit bien de concevoir la tétraévaluation comme un processus d'évaluation au sens très large, comprenant la prise de décision, plutôt que comme une simple procédure de valorisation (monétaire ou non). La société verrait actuellement une concurrence accrue entre des acteurs porteurs d'une conception exclusive de la valeur, ce qui conduirait à une concurrence des valeurs plutôt qu'à une tentative de les harmoniser. Ainsi la démarche est-elle contrastée avec d'autres modes de décision comme le vote majoritaire. Elle suppose de trouver des acteurs ou parties prenantes susceptibles de représenter chaque type de valeur. On a donc affaire à une forme de participation et de délibération un peu particulière dans laquelle les acteurs sont rattachés à une valeur prédéterminée, comme «représentants» de cette valeur. Selon l'auteur, les citoyens représentent logiquement la valeur d'usage, les entreprises la valeur d'échange, les travailleurs la valeur travail et les organisations environnementales la valeur fonctionnelle. Mais si elle présente l'avantage de la simplicité, cette manière de catégoriser les acteurs de la délibération politico-économique n'est pas sans poser problème, nous y reviendrons. 
À de multiples égards, la démarche de F. Gale rappelle celle de l'économie écologique: soutenabilité comme horizon, pluralisme des valeurs, critique de l'économie politique classique et de l'école néoclassique. On est même frappé par la convergence des positions défendues par l'auteur avec celles d'une partie importante de l'économie écologique que l'on nomme aujourd'hui «économie écologique délibérative ${ }^{4} »$. Ce sous-champ de l'économie écologique prend acte d'un ensemble de faits: les individus ne sont pas «rationnels-maximisateurs»; il existe une pluralité de valeurs légitimes qu'une analyse économique monodimensionnelle ne saurait résumer; incertitude radicale, non-linéarités, irréversibilités et complexité caractérisent la plupart des systèmes socioécologiques. De ces prémisses découle la nécessité de traiter les grands enjeux socioécologiques de manière délibérative et réflexive plutôt que de manière positiviste et technocratique. Or il n'apparaît pas que F. Gale pousse la réflexion plus loin (dans cet ouvrage du moins) que celle déjà menée depuis plusieurs décennies en économie écologique par des auteurs tels que Richard Norgaard, Clive Spash, Martin O'Connor, Joan Martínez-Alier, John O'Neill ou Arild Vatn (pour n'en citer que quelques-uns ${ }^{5}$ ). Il semble que seul change le cheminement théorique pour parvenir au résultat, en l'occurrence une articulationdépassement des théories de la «valeur économique». Il aurait été intéressant que des développements soient consacrés aux apports spécifiques de la tétraévaluation par rapport à toutes les autres approches pluralistes-délibératives disponibles. En outre, le détour par les théories de la valeur n'est pas forcément le plus convaincant, ce que nous allons voir maintenant.

Tout d'abord, le traitement réservé aux approches de la « valeur économique » donne l'impression que « la valeur» est considérée comme une substance, dans la lignée d'une part importante de l'économie politique classique et de l'économie néoclassique. Le tournant conventionnaliste n'est donc pas pris, qui reconnaît « la valeur » comme étant essentiellement une construction sociale et qui par conséquent préfère traiter "des valeurs» portées par les individus et les groupes ${ }^{6}$. Désubstantialiser la valeur et rapporter «les valeurs» aux attachements socialement situés des individus et des groupes est l'approche privilégiée par un économiste écologique tel que Joan Martínez-Alier dans ses travaux sur la justice environnementale et les conflits socioécologiques.

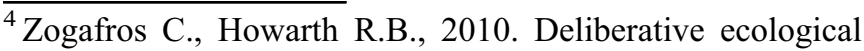
economics for sustainability governance, Sustainability, 2, 11, 3399-3417.

${ }^{5}$ Ceux-ci sont toutefois abondamment cités dans l'ouvrage.

${ }^{6}$ Voir les travaux institutionnalistes en général, l'économie des conventions en particulier, ou encore les travaux issus des valuation studies.
}

Par ailleurs, et comme déjà évoqué précédemment, le parallèle établi entre théorie de la valeur et orientation politique est un peu fragile: pourquoi associer le nationalisme à la valeur d'usage ? La valeur d'échange renverrait au libéralisme, la valeur travail au socialisme, la valeur d'usage à l'étatisme (statism) et la valeur fonctionnelle à l'écologisme. Mais n'est-ce pas sacrifier beaucoup de pertinence et de précision au souci de « faire simple» et à la volonté de préserver le terme «valeur» dans chaque syntagme retenu? Pourquoi ne pas parler de dimensions de soutenabilité? Ou de critères de performance soutenable? Un autre terme, qui charrie moins de présupposés et de confusions, aurait pu être choisi d'autant que l'on ne trouve aucune définition du mot « valeur » dans l'ouvrage. Mais probablement ce choix estil lié au désir de dépasser les visions du monde véhiculées par les théories de l'économie politique, visions souvent résumées comme étant des conceptions de la valeur.

Outre le choix discutable de formuler le débat dans les termes de la «valeur», se pose la question de la légitimité de prôner un «équilibre» entre les formes de la valeur et de mettre réflexivité et délibération au cœur de la quête de soutenabilité. À cet égard, il est intéressant de lire le livre à l'aune des débats francophones contemporains autour de l'économie écologique. Dans leur petit opus de synthèse, Douai et Plumecocq ${ }^{7}$ prennent position pour une économie écologique sociale marxienne qui trancherait en faveur de la valeur travail, dans la lignée des écrits de Jean-Marie Harribey ${ }^{8}$ autour de la valeur, de la richesse et de l'écologie. Cette position se veut critique d'une partie de l'économie écologique délibérative qui donnerait une place trop importante aux valeurs par rapport aux intérêts et serait finalement plus morale qu'une véritable économie politique. À ce titre, l'ouvrage de F. Gale tombe sous les critiques de Douai et Montalban ${ }^{9}$ comme «naïvement» délibératif. On connaît les critiques classiques vis-à-vis des approches multicritères et délibératives: leur caractère trop peu substantiel et trop processuel (le critère de performance réside plus dans le processus que dans le résultat) et donc in fine indéterminé ; leur ouverture à la manipulation par les acteurs les plus puissants ; l'accent mis sur la convergence des vues plutôt que sur le compromis politique; le consensus préféré au compromis issu du rapport de force. Pour certains, comme Jacques Theys,

\footnotetext{
$\overline{7}$ Douai A., Plumecocq G., 2017. L'économie écologique, Paris, La Découverte.

${ }^{8}$ Harribey J.-M., 2013. La richesse, la valeur et l'inestimable: fondements d'une critique socio-écologique de l'économie capitaliste, Paris, Les Liens qui libèrent.

${ }^{9}$ Douai A., Montalban M., 2012. Institutions and the environment: the case for a political socio-economy of environmental conflicts, Cambridge Journal of Economics, 36, 5, 1199-1220, doi: 10.1093/cje/bes046.
} 
l'approche procédurale du développement durable aurait atteint ses limites et montré son inefficacité : «[...] Il y a pour moi une correspondance naturellement étroite entre d'un côté soutenabilité faible, conception procédurale et discours faible sur le développement durable, et de l'autre soutenabilité forte, conception substantive et discours fort. Lorsque je défends la soutenabilité forte, c'est en fait à un retour à la conception substantive du développement durable que je pense ${ }^{10} »$. Si F. Gale n'est pas dupe et ne méconnaît pas les écueils de l'approche délibérative, on aurait souhaité trouver des arguments plus clairs et plus convaincants permettant de penser que la démarche proposée est de nature à promouvoir une approche forte du développement soutenable ainsi qu'une perspective de «transformation» profonde comme celle esquissée par Temper et $a l^{11}$.

Revenons enfin à la prémisse analytique-typologique qui sous-tend le propos: l'auteur suppose que chaque parti politique, autant que chaque acteur convié à la délibération, est l'incarnation d'une seule conception de la valeur, de manière moniste ${ }^{12}$. Or une telle idée est très réductrice et il n'est pas certain que la fiction «syndicat $=$ travail, entreprise $=$ valeur d'échange, parti écologiste $=$ valeur fonctionnelle etc. $\gg$ soit valide empiriquement ni même fructueuse politiquement. On pourrait même soutenir que chemin faisant, les acteurs précités sont conduits à toujours davantage mêler les préoccupations, ou les «valeurs» pour reprendre la terminologie de l'ouvrage: d'où, à titre d'illustration, l'idée de justice climatique qui réussit une percée chez les écologistes contemporains. Dès lors, qui convier? Et selon quelles modalités recueillir les points de vue de chacun? Si l'auteur donne quelques pistes éparses, le lecteur reste tout de même sur sa faim. En outre, si chaque valeur se doit d'être représentée dans le processus de tétraévaluation, la manière dont l'auteur renvoie dos à dos les porteurs de chaque valeur prise individuellement laisse à penser qu'il est structurellement impossible de désigner des (absences de) responsabilités claires dans les problèmes écologiques et sociaux contemporains. Pour filer la métaphore proposée par F. Gale d'une vision «moléculaire » de la soutenabilité,

\footnotetext{
${ }^{10}$ Theys J., Guimont C., 2019. «Nous n'avons jamais été "soutenables": pourquoi revisiter aujourd'hui la notion de durabilité forte?», Développement durable et territoires, 10, 1, doi: 10.4000/developpementdurable.13589.

${ }^{11}$ Temper L., Walter M., Rodriguez I., Kothari A., Turhan E., 2018. A perspective on radical transformations to sustainability: resistances, movements and alternatives, Sustainability Science, 13, 3, 747-764, doi: 10.1007/s11625-018-0543-8.

${ }^{12}$ Le tableau 7.1 p. 169 , qui donne un score aux partis britanniques selon chaque type de valeur, impose toutefois de nuancer notre propos.
}

on se demande parfois par quelle alchimie passer des « valeurs économiques» à « la valeur de soutenabilité».

Les bémols qui viennent d'être énoncés n'enlèvent en rien son intérêt à un ouvrage globalement clair, riche et dont une qualité centrale est selon nous de replacer l'analyse économique dans une perspective profondément pluraliste.

\section{Philippe Roman (ICHEC Brussels Management School, Bruxelles, Belgique et UCLouvain, École des sciences économiques, Louvain-la-Neuve, Belgique) philippe.roman@ichec.be}

\section{Défendre la cause de l'environnement. Une approche organisationnelle}

Nathalie Berny

Presses universitaires de Rennes, 2019, 302 p.

Comment ont évolué les grandes organisations environnementales françaises depuis leur naissance dans les années 1960-1970? Dans cet ouvrage, Nathalie Berny, maître de conférences en science politique à Sciences Po Bordeaux, questionne l'analyse répandue postulant une convergence des modes d'action, des formes organisationnelles et des valeurs portées par les défenseurs de «la cause de l'environnement». En mobilisant une approche organisationnelle sur un objet jusque-là principalement étudié par la sociologie de l'action collective et du militantisme, et dans une moindre mesure, de l'action publique, elle propose une lecture nuancée des grandes évolutions qui ont marqué la croissance et l'institutionnalisation de cinq associations et fondations centrales, sélectionnées pour leur stabilité, leur caractère généraliste et le fait qu'elles soient des interlocuteurs des pouvoirs publics : le WWF, la Ligue pour la protection des oiseaux (LPO), Les Amis de la Terre, France Nature Environnement (FNE) et Greenpeace.

Le premier chapitre pose les bases théoriques qui seront mobilisées pour analyser la trajectoire des organisations de leur création à 2015. Les cinq suivants s'intéressent à des périodes caractérisées par des dynamiques spécifiques. Ainsi, le deuxième chapitre rend compte de la période d'émergence des organisations, marquée par un premier choix de public visé, que ce soit le grand public pour Les Amis de la Terre, les pouvoirs publics pour la LPO, les autres associations pour FNE ou les entreprises pour le WWF et Greenpeace. Le troisième s'intéresse à la période de formalisation, dans les années 1970-1980, entendue comme la consécration d'une compétence distincte dans le temps, par exemple le contentieux juridique pour FNE ou le financement de la conservation pour le WWF. 
Le quatrième chapitre analyse la professionnalisation des organisations à partir de la fin des années 1980, dans un contexte de baisse de l'activité militante et de précarité financière. Le cinquième porte sur le processus d'institutionnalisation partielle, "c'est-à-dire l'intégration inégale des compétences qui caractérise les structures françaises » (p. 12) au début des années 2000. Enfin, le sixième et dernier chapitre pose la question de l'impact du «tournant collaboratif des politiques publiques», en particulier pendant et après la présidentielle de 2007 et le Grenelle de l'environnement.

L'originalité de l'ouvrage tient d'abord à sa perspective pragmatiste, qui «postule l'inventivité des acteurs face aux situations qu'ils doivent démêler» (p. 10) et organisationnelle, en s'intéressant à l'adaptation des organisations à leur environnement. Au centre de cette démarche théorique, la notion de «compétence distincte» permet de saisir dans un même geste deux explications souvent séparées : par le contexte et par la forme organisationnelle. Autrement dit: «Une organisation se construit dans le temps au terme d'un processus d'institutionnalisation qui résulte de l'interaction entre pressions externes et besoins internes ». (p. 25)

Synonyme de modération du discours et de collaboration accrue avec les pouvoirs publics en sociologie des mouvements sociaux, et donc d'homogénéisation, le phénomène central qu'est l'institutionnalisation est ici défini comme une singularisation des organisations. Il s'agit alors de montrer comment ce phénomène se matérialise différemment dans chaque organisation, à rebours de l'idée répandue d'une convergence et d'une hybridation des modes d'action et des discours. Dans tous les cas, l'ouvrage montre que l'institutionnalisation reste partielle et fragile, même pour des organisations comme le WWF. Sur la période étudiée (des années 1960 à 2015), « Si la convergence est manifeste sur le plan des modes d'action, elle ne l'est pas nécessairement sur celui de l'organisation interne et des valeurs. » (p. 254)

Cette comparaison se base sur un travail empirique conséquent, sur archives et des entretiens, ce qui permet de retracer minutieusement la trajectoire des cinq organisations retenues. La perspective longitudinale rend possible l'analyse du processus de développement des organisations en regard des attentes de leurs publics externes, d'une part (qu'elles soient réelles ou imaginées), et de leur identité et leur histoire, d'autre part.

L'ouvrage permet ainsi de nuancer, et parfois de contester, des catégories binaires trop souvent mobilisées pour qualifier les mouvements environnementalistes, que ce soit opposition/dialogue ou contestation/réforme concernant les modes d'action, écologiste/conservationniste, participation forte des bénévoles ou non, ONG internationales/associations nationales, etc. Plutôt que d'analyser les évolutions que connaissent les organisations à l'aune de leur appartenance à ces catégories, on voit ici tout l'intérêt de l'approche pragmatiste qui permet de saisir avec finesse le travail d'expérimentation et d'allers-retours stratégiques qui caractérise la vie des organisations environnementales. Ainsi, une organisation comme Les Amis de la Terre, que l'on classe volontiers dans les catégories écologiste et contestataire, a pu développer une activité de conseil à destination des entreprises et des collectivités locales au début des années 1990 avant de revenir vers une activité plus militante et plus proche des groupes locaux, rejetant par là même la coopération avec le secteur privé.

Ces «crises de sens» régulières sont souvent analysées sous l'angle de la tension entre bénévoles et salariés, ou entre le rôle d'expert et celui de militant. $\mathrm{N}$. Berny montre que la professionnalisation implique aussi une «compétition entre les savoirs pertinents, portés par des professions différentes, pour mener à bien les missions de l'organisation» (p. 263). Cette concurrence concerne «les problèmes et solutions pertinentes relevant des états naturels, les dispositions des publics susceptibles d'avoir un impact sur eux et le maintien de l'organisation » (p.265-266). Sur ce dernier aspect, la partie portant sur la période de croissance qu'a été la première moitié des années 2000 montre comment les organisations se sont approprié différemment les méthodes de management et amène l'auteure à relativiser leurs effets négatifs sur «l'esprit associatif».

Au-delà des cinq organisations, l'ouvrage permet d'actualiser les données disponibles sur le milieu associatif environnemental. C'est notamment un des premiers à s'essayer à un bilan des conséquences du Grenelle de l'environnement et du « tournant collaboratif des politiques publiques» sur les associations environnementales. Les organisations ont été amenées à expérimenter de nouveaux modes d'action et à collaborer sous la pression des pouvoirs publics et des organisations internationales alliées. Un effet particulièrement intéressant est le fossé créé par la consultation entre les experts, représentant habituellement l'organisation, et les décideurs (direction et élus associatifs), qui ont monopolisé le travail de négociation avec le politique. La convergence est toutefois temporaire puisqu'on observe un retour à une spécialisation, certes relative, dans un contexte de compétition accrue pour les ressources financières et de changement politique marqué par un reflux de l'intérêt pour les questions environnementales. Ce retour aux compétences spécifiques se fait de manière plus ou moins évidente, les organisations n'ayant pas toutes développé les mêmes capacités de résilience.

Cet ouvrage, en prenant dès le départ des options théoriques et empiriques fortes et originales, porte un regard nouveau sur le développement des grandes associations et fondations environnementales françaises. 
Mais au-delà du cas étudié, il questionne avec profit des processus et des catégories qui restent trop souvent à l'état de postulat, que ce soit l'homogénéisation des formes organisationnelles, l'évidence du lien entre professionnalisation et institutionnalisation, ou l'égalité des organisations face à l'ouverture ou la fermeture de la structure des opportunités politiques.

\section{Malo Herry (Université de Rennes 1, UMR6051 Arènes, Rennes, France) maloherry@gmail.com}

\section{Hommes-Milieux. Vers un croisement des savoirs pour une méthodologie de l'interdisciplinarité}

Adélie Pomade (Ed.)

Presses universitaires de Rennes, 2018, 222 p.

Hommes-Milieux est né suite au colloque international qui s'est tenu en mai 2018 à l'Université de Rennes 1. Il n'en est pas l'aboutissement ou la conclusion mais constitue plutôt, c'est là toute son originalité, une étape supplémentaire à une réflexion sur le thème «travailler ensemble ». Dans cet ouvrage très novateur, l'interdisciplinarité se construit au fil des pages dans un agencement progressif qu'analyse et synthétise Adélie Pomade (Droit, Université de Bretagne-Occidentale), la coordinatrice scientifique. L'auteure questionne d'emblée l'importance de « travailler ensemble», organisation complexe à mettre en œuvre. Alors comment procéder? Elle va élaborer une grille de lecture de textes écrits par divers auteurs autour du lien hommes-milieux qui sera l'ancrage commun auquel le plus grand nombre pourra s'exprimer selon sa propre discipline. Pour ancrer plus finement encore les textes, elle va décliner cette relation hommes-milieux en trois : celle qui concerne les hommes et les territoires (comment les hommes s'inscrivent dans les territoires ?), les hommes et la biodiversité (comment les hommes impactent la biodiversité ?) et l'homme et la nature (comment les hommes vivent leur lien à la nature?). Une construction qui va donc s'analyser progressivement sous trois niveaux; spatial, biologique et spirituel. Comment les diverses disciplines s'emparent-elles de ces relations ? Comment les déclinentelles?

Dans la première partie, les auteurs sollicités ont rédigé leurs textes en suivant plusieurs consignes : il fallait choisir un objet d'étude précis selon leurs intérêts scientifiques, exposer une problématique particulière soulevée parmi les trois évoquées plus haut, expliciter la perception d'une des relations choisies selon la discipline, exprimer les lacunes rencontrées au sein des disciplines, tenter de dépasser les obstacles (quelles méthodes ou données mobiliser?) et enfin formuler des pistes pour en sortir.
Les auteurs se succèdent sous un angle très libre: dans la partie « Hommes-territoires », Chloé Vlassopoulos se focalise sur «Les migrations environnementales: un problème imprécis et déterritorialisé?» (p.31), Sébastien Boillat aborde «Le rôle de la microtoponymie dans la reconnaissance des savoirs autochtones en gestion territoriale rurale» (p.43), Johan Ozswald, le «Suivi des enjeux de conservation par la résilience des écosystèmes en contexte forestier tropical humide» avec l'exemple de l'Amazonie (p.61). Dans la partie «Hommes-biodiversité», après une introduction de Nathalie Hervé-Fournereau qui discute du contexte de la biodiversité, Benjamin Singer propose un texte qui traite d'un « régime international sans noyau ? le cas des forêts» (p. 83). Catherine Aubertin interroge «Le protocole de Nagoya à l'épreuve de la recherche sur la biodiversité » (p.99), Alexandra Aragão expose les «Symbioses disciplinaires autour du droit en faveur de la biodiversité» (p.113). Enfin, dans la partie «Hommes-nature», Nicole Mathieu se demande en introduction «quelle interdisciplinarité [mobiliser] pour construire collectivement l'exploration de la relation hommes-milieux?» (p. 129). Denis Couvet, dans cette même et dernière partie, traite de la biodiversité entre épistémologies et conceptions du monde (p. 133), Priscilla Cardoso Rodrigues du «rôle des savoirs traditionnels indigènes dans la théorie des services écosystémiques» (p.149) et Christoph Eberhard, de «L'humain, la nature et au-delà : dialogues» (p. 165). Ainsi se succèdent, au fil des chapitres, des objets, des orientations, des questionnements divers de chacune des disciplines convoquées selon des méthodes de travail multiples proposées au gré des ancrages choisis.

À ce stade de l'ouvrage, le lecteur, même s'il apprécie les différents textes qui renseignent sur des champs très divers, est un peu perdu et peut renoncer à continuer sa lecture. La liberté laissée aux auteurs a sa richesse, sa logique propre mais elle interroge aussi sur le lien potentiel entre les différents articles. Doit-on, peut-on en faire une lecture séparée ? Doit-on, peut-on continuer une lecture suivie d'un texte à l'autre? Le lecteur entre alors dans une phase d'interrogations multiples.

Et pourtant c'est là que réside la grande originalité du livre, dans l'exercice même que va opérer A. Pomade. Effectivement, l'examen des différents récits est le point de départ d'un travail méthodologique, minutieux et profond qu'elle va initier dans une seconde partie du livre pour saisir les complémentarités, les divergences, les raisonnements connexes, les perspectives de chaque texte et ainsi envisager la complémentarité des modes de questionnement ou, à l'inverse, les divergences et les cloisonnements.

Cette deuxième partie, intitulée Hommes-milieux: analyse méthodologique, est en définitive le commencement du livre et avec force et prouesses, l'auteure nous 
plonge dans une lecture seconde des écrits qu'elle revisite avec brio dans une synthèse particulière. Que nous apprend-elle?

Dans un premier chapitre, elle reprend les trois relations et regarde chacune d'entre elles en essayant une incroyable analyse. Dans la relation hommesterritoires, elle remarque deux aspects qui se retrouvent dans les chapitres de la première partie consacrée à ce sujet. Le premier est «l'attachement au lieu», en tant que « reconfiguration » (migrations humaines) ou pris dans une dimension "spirituelle» (lien de l'être humain avec l'espace considéré). Le second est la prise en compte de l'homme dans la relation, notamment par «l'intensité ». Une intensité comme un «attachement spirituel», par exemple, ou comme une "appropriation du sol». A. Pomade nous montre à quel point les disciplines se parlent entre elles, ce qui permet d'aller vers « une logique multi-niveaux montant en complexité $»$ pour reprendre ses propres termes. Dans la partie hommes-biodiversité, elle insiste sur «les divergences d'intérêts et de perceptions à son égard " mentionnées dans les textes des différents auteurs. Si chacun exprime l'importance d'une diversité d'acteurs en lien avec la biodiversité, l'intensité de la relation entre les deux est aussi clairement signalée. L'auteure nous explique ensuite comment chacun des auteurs recourt à des champs disciplinaires différents pour exprimer toutes les tensions récurrentes autour de la biodiversité. Dans le chapitre Hommes-nature, elle évoque «la diversité des perceptions et des conceptions des Occidentaux ou des peuples indigènes $»$. Elle signale, par exemple, comment deux modèles de comportement à l'égard de la nature sont retrouvés indirectement dans les trois textes et peuvent conditionner l'attachement de l'être humain à la diversité biologique. Le premier modèle, « valeurs-croyance-normes », constitue un cadre méthodologique important qui permet de «caractériser les représentations des individus ». Le second, «théorie des représentations sociales», permet d'étudier les liens existants dans les représentations sociales et les comportements. Ces deux modèles se retrouvent dans les concepts dictés par les auteurs, notamment dans ceux «d'unité nature », de « conception du monde » ou « dans le dialogue macrocosme-microcosme». A. Pomade conclut que la nature est ainsi envisagée au fil des textes de manière « intégrée » et « comme un Tout dépassant la somme des parties». Les termes "symbioses», "cosmovision», partagés par les auteurs, suggèrent que cette vision holiste est bien celle qui conduit «au développement mental, spirituel, intellectuel et philosophique de l'homme». Elle termine en ajoutant « qu'au sein de ce Tout, les dimensions s'harmonisent en interrelations constantes entre les éléments » (humains-non humains) [p. 195].

Dans un second et dernier chapitre, A. Pomade recherche les « convergences méthodologiques communes » aux trois relations et revient sur le rapport au temps, que l'on retrouve dans tous les textes, à l'espace, ou encore à des concepts transdisciplinaires (services écosystémiques, l'éthique) exploitables par diverses disciplines. Elle évoque ensuite les défis communs comme celui de la fragilité de la transmission, l'idée d'être toujours en capacité de garder une certaine cohérence dans le discours et une certaine lisibilité des écrits, de savoir en permanence articuler les enjeux pluridisciplinaires ou encore comment en restant trop à la périphérie des disciplines, dépasser une plurisectorialité peu intéressante en soi, pourquoi redouter la complexification des objectifs des disciplines et enfin comment aller vers une réelle et sincère hybridation des sciences. Elle termine en évoquant les différents types de relations entre les disciplines. Est-ce que certaines sciences peuvent en remplacer d'autres? Est-ce que certaines peuvent être les garde-fous d'autres ? Certaines pourraientelles remplacer la relation de la recherche à la nature ? Que peuvent-elles prendre chacune des autres disciplines?...

Pour conclure, vous l'aurez peut-être compris, ce livre ne se raconte pas, il se découvre au fur et à mesure qu'il se lit. Il traduit, et c'est bien sa force, le travail méthodologique que l'auteure nous propose mais aussi celui que nous pouvons nous exercer à construire nous-mêmes. Il n'y a donc pas une seule, mais de multipes lectures possibles, avec des points de départs variés. A. Pomade nous aide en donnant l'exemple et en nous livrant une méthodologie pour construire l'interdisciplinarité. Si la sienne est admirable, elle invite aussi à bien d'autres. À nous de nous en saisir si nous le souhaitons. Ce livre est bien à la fois un essai méthodologique et un guide pour aborder et mieux comprendre l'interdisciplinarité.

Nathalie Frascaria-Lacoste (AgroParisTech, UMR8079 ESE, Orsay, France) nathalie.frascaria@u-psud.fr

\section{Paroles de chercheurs. Environnement et interdisciplinarité}

Évelyne Brun, Jean-François Ponge, Jean-Claude Lefeuvre Quæ, 2018, 126 p.

Cet ouvrage, dont le volume ressemble plus à celui d'un essai, présente de toute évidence un intérêt pour qui s'interroge sur le sens de l'interdisciplinarité et l'utilité de la pratique interdisciplinaire. Et a fortiori pour les lecteurs des «Recherches et débats interdisciplinaires » de Natures Sciences Sociétés. C'est d'abord pour son objectif: «mettre en lumière certains aspects du chemin parcouru par des chercheurs qui ont accepté de se fondre dans des opérations de recherche avec une obligation 
d'interdisciplinarité » et, ce faisant, «mettre en évidence des manières d'agir développées par ces chercheurs pour définir leur "posture"»(p.11). Comme annoncé dès l'introduction, il s'agit de poursuivre l'entreprise initiée par Évelyne Brun qui donna lieu à une publication dans $N S S^{13}$. Comprendre et qualifier l'interdisciplinarité «à la française » de la recherche sur l'environnement en est le but.

Pour la rédaction de l'ouvrage, l'équipe réduite à deux des auteurs de l'article de 2007, Évelyne Brun (ingénieure, CNRS) et Jean-Claude Lefeuvre (écologue, professeur émérite du Muséum national d'histoire naturelle [MNHN]), s'est adjoint l'écologue des sols Jean-François Ponge suite à la disparition de Jean-Marie Betsch (écologue, MNHN) en 2013. Elle a prolongé patiemment le travail initial avec l'ambition de restituer les leçons de cette longue expérience autoréflexive menée par et autour d'un groupe de 19 scientifiques, nés entre 1940 et 1960, se rattachant à des départements distincts (sciences de l'univers, sciences de la vie, sciences de l'ingénieur, sciences humaines et sociales) et des disciplines différentes (écologie, botanique, géographie, ethnologie, physique, sociologie, droit) qui tous avaient été impliqués dans les grands programmes interdisciplinaires mis en œuvre dans les années 1970 et jusqu'à la fin des années 1990.

Rendre visibles et compréhensibles l'histoire et les particularités des pratiques de l'interdisciplinarité en France éclaire et complète incontestablement la quête épistémologique que NSS n'a cessé de mener depuis sa création peu après la publication des Passeurs de frontières ${ }^{14}$. Il suffit, pour le prouver, de renvoyer le lecteur aux dossiers Interdisciplinarité et formation à l'interdisciplinarité publiés dans la revue ainsi qu'aux ouvrages issus des animations de l'association NSS Dialogues: Modélisation et interdisciplinarité $^{15}$, Interdisciplinarité entre Natures et Sociétés ${ }^{16}$. L'interrogation sur la valeur heuristique et l'utilité sociale de l'interdisciplinarité dans le contexte actuel de la politique de la recherche est aujourd'hui particulièrement présente chez

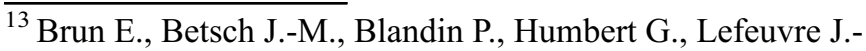
C., Marinval M.-C., 2007. Postures des scientifiques et interdisciplinarité dans le champ de l'environnement, Natures Sciences Sociétés, 15, 2, 177-185, doi: 10.1051/nss2007045.

${ }^{14}$ Jollivet M. (Ed.), 1992. Sciences de la nature, sciences de la société. Les passeurs de frontières, Paris, CNRS Éditions.

${ }^{15}$ Mathieu N., Schmid A.-F. (Eds), 2014. Modélisation et interdisciplinarité. Six disciplines en quête d'épistémologie, Versailles, Quæ, collection Indisciplines.

${ }^{16}$ Hubert B., Mathieu N. (Eds), 2016. Interdisciplinarités entre Natures et Sociétés. Colloque de Cerisy, Bruxelles, P.I.E. Peter Lang.
}

les jeunes thésards et postdocs ${ }^{17}$. Ils trouveraient sans doute des lumières si ce n'est des réponses à leurs doutes en prenant connaissance des points de vue de cette génération de scientifiques qui les a précédés.

Mais, pour qui prône et pratique l'interdisciplinarité, l'intérêt de cet ouvrage ne se limite pas à l'objectif général que nous venons de présenter. La démarche choisie pour l'atteindre mérite aussi d'être soulignée de même que son titre peu académique et plein de promesses: Paroles de chercheurs. En effet, avec pour hypothèse principale que tout au long de sa carrière un chercheur agit à partir d'invariants caractéristiques de sa posture, le dispositif de recherche s'appuie principalement sur des entretiens longs voire récurrents, conduits librement avec une qualité d'échange entre interviewer et interviewé due à la connaissance partagée des programmes environnement. Pour les auteurs, l'analyse de ces entretiens doit permettre de repérer les éléments constitutifs de la posture du chercheur - le noyau, la plasticité et l'empreinte -, tandis que celle de ces «dires» doit faire émerger des types de «postures». L'importance accordée aux paroles de chercheurs est identique, là encore, à celle que NSS donne à la rubrique «Entretien » : comprendre les différences de positionnement sur l'interdisciplinarité dans les autres courants interdisciplinaires qu'ils soient francophones ( $c f$. entretien avec Edgar Morin ${ }^{18}$, avec Isabelle Stengers $\left.{ }^{19}\right)$ ou non $\left(c f\right.$. Pohl $\left.^{20}\right)$, mais aussi au sein de la mouvance (pour exemple Georges Bertrand) ou en son centre (Jean-Marie Legay, Jean-Pierre Deffontaines...)

\footnotetext{
${ }^{17} \mathrm{Cf}$. le séminaire sur les thèses interdisciplinaires à l'Inra. Il s'agit du «Projet BASC-Our», équipe dirigée par Pierre Chassé, Sarah Cogos et Timothée Fouqueray. Ils ont organisé le 2 avril 2019 à AgroParisTech une journée confrontant des « experts » de l'interdisciplinarité (Nicole Mathieu, Nathalie Machon et Patrick Steyaert) à de «jeunes» thésards préoccupés par la compréhension des enjeux d'une thèse interdisciplinaire ainsi que de la pratique de l'interdisciplinarité évaluée par rapport à ses méthodes.

${ }^{18}$ Egdar Morin : le «contrebandier» d'une pensée complexe, propos recueillis par Nicole Mathieu, Alfredo Pena-Vega et Marianne Cohen, Natures Sciences Sociétés, 4, 3 (1996), 250-257, www.nss-journal.org/articles/nss/pdf/1996/03/ nss 19960403p250.pdf.

${ }^{19}$ Discipline et interdiscipline : la philosophe de «l'écologie des pratiques » interrogée. Entretien avec Isabelle Stengers en présence de Pierre Verstraeten, propos recueillis par Nicole Mathieu, Natures Sciences Sociétés, 8, 3 (2000), 51-58 ( $1^{\text {re }}$ partie) et Natures Sciences Sociétés, 8, 4 (2000), 57-63 ( $2^{\mathrm{e}}$ partie), www.nss-journal.org/articles/nss/pdf/2000/04/ nss20000804p57.pdf.

${ }^{20}$ Pohl C., 2001. How to bridge between natural and social sciences? An analysis of three approaches to transdisciplinary from the Swiss and German field of environmental research, Natures Sciences Sociétés, 9, 3, 37-46, doi: 10.1016/S1240-1307 (01)80047-9.
} 
Aussi, lors de la première lecture, l'ouvrage apparaît méthodiquement construit dans une écriture claire. On pourrait presque dire qu'il a été conçu comme un manuel destiné à faire connaître à des étudiants ou à toute personne curieuse de l'approfondir ce «moment», sorte d'apogée du rapport entre recherche, environnement et interdisciplinarité.

La préface de Serge Bahuchet du MNHN donne le ton. Il rend hommage à «Évelyne Brun et au regretté Jean-Marie Betsch, tous deux membres du bureau du $\mathrm{PEVS}^{21}$ » pour avoir animé ce groupe de réflexion et mené à son terme «ce travail lent et méticuleux» qui contribue à «réunir les sciences disciplinaires» pour qu'elles reprennent leur place dans la société civile (p. 3-4). Comme en écho, une citation lumineuse de JeanMarie Legay (p. 9) définit l'interdisciplinarité qui n'est ni un «principe épistémologique, ni une mode, ni une contrainte institutionnelle » car «c'est dans les questions qu'il faut nous débattre ». Après cette entrée en fanfare, les remerciements qui donnent des clés pour repérer les témoins qui ont été volontairement rendus anonymes et l'introduction de facture classique, l'ouvrage déroule sept chapitres de taille inégale. Les deux premiers chapitres de 8 pages chacun tentent un récit historique en deux périodes, l'une où la question environnementale provoque l'émergence de la recherche interdisciplinaire au sein de l'Etat français, la deuxième où «la communauté s'organise» en comités, programmes de recherche et appels d'offres pilotés par le CNRS. Cette histoire de la place que la politique publique donne à l'interdisciplinarité dans un lien avec la «demande sociale», elle-même liée à «l'environnement», est illustrée par des exemples de comités (Le SEAH Systèmes écologiques et actions de l'homme p.21) et d'appels à propositions (celui du SEAH daté de 1995 p. 27-28). Il en ressort une interprétation de cette politique comme volonté de faire travailler ensemble des chercheurs de différentes disciplines «hors de tout contexte hiérarchique » qui s'interrogent sur «l'utilité de leurs savoirs et sur leur implication dans les problèmes mondiaux non résolus» (p.29-30). Outre le fait que l'ancêtre de la recherche interdisciplinaire promu par la Délégation générale à la recherche scientifique et technique (DGRST) - Plozévet - soit passé sous silence dans ces deux chapitres, il serait intéressant de les confronter à d'autres interprétations de l'histoire de la recherche interdisciplinaire à la française mêlant la vision institutionnelle (d'en haut) à celle des chercheurs de base (d'en bas).

Pour présenter la méthode, les outils d'analyse des «porteurs de l'interdisciplinarité », le chapitre 3 n'a luimême que 3 pages. On en retient peu d'éléments

\footnotetext{
${ }^{21}$ PEVS : programme Environnement vie et sociétés du CNRS.
}

nouveaux par rapport à l'introduction. D'abord que sur les 21 collègues sollicités, 19 (17 hommes et 2 femmes) ont accepté ; ensuite, que tous les entretiens obtenus par "un processus itératif»ont été analysés «pour faire émerger une "typologie des postures" »; que la question principielle "Qu'est-ce qui dans votre carrière vous a amené à vous intéresser à l'interdisciplinarité ?» a entraîné des réponses qui feront le socle de l'analyse; enfin, qu'il y a eu recours à des approches statistiques par l'établissement d'une «matrice à dires d'expert» qui a permis de "pratiquer une analyse posturale» qu'une «analyse lexicale» a complétée.

Le chapitre 4, «Paroles de chercheurs en environnement» (32 pages), forme en quelque sorte le cœur de l'ouvrage par une présentation à peine ordonnée des réponses faites par les interviewés à la question initiale. Quelques titres («Ils répondent à la demande sociale», «Confiance ou excellence», «Théorie ou pragmatisme», «La notion de réseau ou de clan», «Liberté, réseaux et clans : existe-t-il des groupes harmonieux?», «Confiance en soi, optimisme obligatoire mais doute $», ~ «$ Comment sont vécues les structures de recherche et l'appartenance à une discipline?», «Le temps long et les outils pour communiquer », "La prise de risque ou la peur du risque »)... ne constituent pas une grille de lecture explicite des paroles des chercheurs sous forme de longues citations qui attirent le lecteur tant elles sont riches de phrases clés, sorte de devises diverses et parfois contradictoires. Toujours dans le chapitre 4, la partie intitulée «La recherche interdisciplinaire » semble ouvrir un temps de synthèse dans cette abondance de paroles passionnantes. Mais là encore, les passages rédigés par les auteurs de l'ouvrage arrivent difficilement à détourner le lecteur des citations qui différencient l'écologue Dominique de Guillaume le physicien, de Paul le mathématicien écologue ou de l'intéressant Lucien le sociologue, etc., qu'il ne peut que préférer à ce que dit Bernard l'économiste ou André le géographe... Peu importe que l'on en tire les « limites de la pratique » interdisciplinaire ou les «Difficultés relationnelles? Dans certains cas c'est difficile, dans d'autres c'est idyllique », on ne peut s'empêcher de rechercher sous les citations d'un même nom la cohérence et la justesse de pensée d'une personne dont on se sent proche ou dont on aimerait approfondir l'éthique épistémologique et la démarche dans l'engagement pour le «Transfert des connaissances», ou l'enseignement et la position sur l'« Expertise »... Si bien que l'esquisse de conclusion de ce chapitre met en avant l'empreinte laissée sur les chercheurs impliqués dans le comité scientifique par un « effet de groupe » et laisse le lecteur sur sa faim en ce qui concerne les autres éléments constitutifs de la posture que les auteurs prétendaient faire émerger des entretiens.

Mais les chapitres 5, «Rôles des porteurs de l'interdisciplinarité », et 6 , «Posture et réussite de l'interdisciplinarité », corrigent incontestablement cette impression. 
En menant en parallèle la méthode des citations et une démarche classique de modélisation, trois types de posture sont identifiés : le « concepteur ou architecte », le « chef de projet ou pilote» et le «chercheur de terrain ou acteur». Quelques citations de scientifiques illustrent chacun des trois types de cette typologie qui semble plus liée à un rôle qu'à une trajectoire de pensée. Puis une longue série de citations non classées dans la typologie précédente est censée aider à instruire les trois mots qui constituent «les postures $»$ : l'empreinte déjà définie plus haut ; la plasticité, qui renvoie à la capacité d'adaptation et de « transformer ce qui pour certains seraient des contraintes en une nouvelle base de travail ou d'organisation » (p. 92); enfin le noyau, ce qui ne peut être modifié et qui a construit le chercheur. Mais les cinq pages de cette série de citations qui pourtant regorgent d'expressions lumineuses de certains scientifiques dont on entrevoit la personnalité, voire la "personne», n'éclairent guère ni sur ce qui fait noyau, plasticité et empreinte, ni sur leur combinaison qui différencie les postures. C'est finalement une analyse en composante principale qui apporte une clarification. Elle montre que le principal facteur structurant et la manière dont est pratiquée l'interdisciplinarité ont à voir avec la discipline principale de l'intéressé: entrée «naturelle» dans l'interdisciplinarité pour les «acteurs» quand ils travaillent sur les questions de société (sciences humaines et sociales), entrée «brutale» quand on est « constructeur de projet » et lorsqu' on appartient aux sciences dites «dures» (sciences de l'univers). La vérification poussée de la relation disciplinaire avec la pratique de l'interdisciplinarité semble contredire ce résultat si bien que les auteurs concluent cette analyse en ces termes: «La décision de pratiquer semble donc relever d'une démarche personnelle alors que la façon dont on est en relation avec les chercheurs d'autres disciplines est fortement marquée par celle à laquelle on appartient» (p. 106). Plus convaincante à nos yeux est l'analyse lexicale conduite par les auteurs qui permet de passer du classement en trois groupes de mots les plus significatifs aux trois types de posture annoncés au début de ces chapitres consacrés aux résultats de l'entreprise.

Le chapitre 7, «Interdisciplinarité pour l'environnement. Quelles perspectives? », forme avec la conclusion de l'ouvrage (sept pages en tout) un ensemble de remarques sur les conditions d'une pratique interdisciplinaire créative de connaissances nouvelles et socialement utiles. On ne peut qu'y adhérer tant l'accent est mis sur la nécessité d'un temps long et de liberté pour les chercheurs curieux et décidés à instruire questions et problèmes irrésolus entre natures et sociétés.

Mais une fois le livre refermé, le besoin se fait sentir de le relire une ou deux fois encore pour en tirer plus qu'il n'apporte. C'est sans doute parce que son écriture ne convient pas à l'exercice d'une démonstration et rend difficile la compréhension des réponses apportées aux deux questions de sa problématique: quelles sont les postures des chercheurs qui s'engagent dans l'interdisciplinarité et comment les identifier? En quoi ces postures participent-elles de la réussite ou de l'échec des programmes et des pratiques interdisciplinaires? Mais la difficulté provient surtout de la fragmentation des paroles des chercheurs, qui de mon point de vue, contredit le repérage des "postures» archétypales qui émergent des «personnes» et du récit de leur engagement dans une situation de recherche. Quoi qu'il en soit, cet ouvrage débordant de véritables perles appelle à une discussion collective de son contenu pour une histoire évaluée de l'interdisciplinarité.

\section{Nicole Mathieu \\ (Directrice de recherche honoraire, CNRS, UMR7533 Ladyss, Nanterre, France) Nicole.Mathieu@univ-paris1.fr}

\section{Leurre et malheur du transhumanisme}

Olivier Rey

Desclée de Brouwer, 2018, 193 p.

Naguère, le transhumanisme était paré d'un certain mystère. Rejeton atypique de la contre-culture californienne des années 1960 , il pouvait passer pour un laboratoire d'idées potentiellement fécondes une fois débarrassées de leurs outrances prophétiques ou de leurs exagérations positivistes. Ou bien, au contraire, tenu pour la dernière utopie glaçante élaborée par des savants fous et par des ingénieurs libertariens prêts à liquider «notre-commune-humanité » au nom d'un progressisme devenu incontrôlable, il faisait figure de repoussoir et d'usurpateur : rien n'évoquait en lui l'humanisme dont il se réclamait pourtant. Lourd de toutes sortes de menaces, il relevait, comme mouvement, du cordon sanitaire et ses partisans comme individus, de la psychiatrie. Les choses ont récemment changé. En premier lieu, certains transhumanistes ont compris qu'ils n'ont rien à gagner à se présenter comme des prophètes de la mort de l'homme, de même qu'il y a eu des prophètes de la mort de Dieu. Ils ont donc « réduit la voilure » et proposent des objectifs en apparence modestes et réalisables : vivre plus longtemps en meilleure santé. On pense ici à la stratégie de l'Association Française Transhumaniste qui défend une version assez bénigne du transhumanisme où il est «seulement» question d'améliorer les caractéristiques physiques et mentales humaines, dans une perspective modérée, raisonnable et inclusive, qui ne laisserait personne de côté: les bienfaits du progrès technique ruisselleraient jusqu' aux derniers de cordée et l'environnement lui-même s'en trouverait rehaussé. En second lieu, plusieurs auteurs, prenant au sérieux les thèses des transhumanistes, ont essayé de reconstituer la pensée de ceux-ci et de l'inscrire dans des traditions philosophiques reconnues. On pense ici à Gilbert Hottois, philosophe et 
universitaire belge qui soutient que le transhumanisme, par sa capacité à envisager le caractère abyssal de la condition humaine, est bel et bien un humanisme.

Olivier Rey, polytechnicien, chercheur au CNRS et membre de l'Institut d'histoire et de philosophie des sciences et des techniques connait sans doute ces développements récents du débat, mais considère que les choses doivent être, en un sens, abordées comme si rien, ou du moins peu de choses, n'avait été fait. Il faut démêler, dans le projet transhumaniste, ce qui mérite d'être pris au sérieux et ce qui relève de la propagande pure et simple. La propagande a «pour mission de masquer ce à quoi elle s'emploie à frayer la voie» (p. 9-10). Ce qui est sérieux est le fait que le transhumanisme, en dépit de toutes les critiques qu'il a suscitées et suscite encore, continue de fasciner. Il est nécessaire d'interroger la nature de cette fascination.

L'ouvrage de O. Rey est donc construit selon ces deux axes. Un premier chapitre montre à l'œuvre la propagande transhumaniste ou, en d'autres termes, son «idéologie » (le terme figure p. 51) puisque l'on a affaire à une doctrine qui masque ce qu'elle exprime et prépare. Les deux chapitres suivants mettent en évidence ce qui est apte à séduire dans une telle idéologie.

Les idéologies se signalent assez rarement par leur rigueur. Le premier chapitre va donc, non sans une certaine jubilation, montrer ce que la propagande transhumaniste a d'incohérent et d'insidieux à la fois. À cet effet, l'auteur se rapporte au célèbre «chaudron » freudien. Il s'agit, rappelons-le, de la réponse de l'emprunteur d'un chaudron à son voisin qui l'accuse de lui avoir rendu endommagé : «D'abord, quand je t'ai rendu ce chaudron, il était en parfait état; ensuite, quand tu me l'as prêté, il était déjà troué ; d'ailleurs, en fait, je ne te l'ai jamais emprunté ». Le côté farce de l'anecdote consiste en ceci que l'on a affaire à un système de défense dont chaque élément est plausible s'il est pris séparément. Mais si on les rassemble, le système s'effondre car les arguments s'excluent mutuellement. C'est une telle « logique » que O. Rey estime à l'œuvre dans la propagande transhumaniste. Elle a pour objet «de convaincre les populations de donner leur adhésion pleine et entière» (p. 17) aux innovations technologiques susceptibles de réaliser une amélioration radicale de la condition humaine. La propagande transhumaniste annonce alors toutes sortes d'avantages que cette révolution va apporter: intelligence artificielle d'une puissance extraordinaire, libération d'une chiche dotation biologique léguée par une marâtre nature, immortalité. Aux sceptiques qui objecteraient que les bienfaits technologiques ont toujours un coût et que ces bénédictions doivent bien s'accompagner de quelques désavantages, on répondra que ce qui se fait aujourd'hui et se fera demain n'est que le prolongement de ce qui s'est toujours fait depuis que les êtres humains utilisent des techniques, c'est-à-dire depuis que l'humanité existe.
Enfin, à ceux qui resteraient incrédules malgré tout, on rétorquera qu'il est vain de s'opposer à une telle révolution, qui est inéluctable. Une formule brillante résume les choses : «[...] quand le discours de l'enchantement transhumaniste peine à convaincre et que le discours de la continuité transhumaniste laisse perplexe, c'est le discours de la fatalité transhumaniste qui prend le relais » (p. 26).

Cette idéologie a une fonction et un sens bien précis : il devient de plus en plus difficile de dissimuler que le monde tel qu'il va ne va pas bien. L'inflation des promesses vise donc à restaurer la confiance en un progrès dont on a de plus en plus l'impression qu'il ne conduit pas vers un avenir toujours meilleur mais instaure un présent de ruines et de dévastations. Ces promesses tendent aussi à détourner l'attention de ceux qu'elles révulsent: en conjuguant au futur l'horreur d'un monde totalement transhumanisé, elles rendent relativement indulgent envers un monde en voie de transhumanisation.

Les deux chapitres suivants proposent une explication de la séduction des thèses transhumanistes aux yeux de nombre de nos contemporains. Un point essentiel de l'argumentation de l'auteur dans ces deux chapitres est que cette séduction ne dépend pas d'un goût immémorial pour l'utopie ou pour les promesses d'un avenir radieux. Elle renvoie, au contraire, à l'état de diminution qui est aujourd'hui celui de l'être humain ainsi qu'à la vulnérabilité de la pensée contemporaine face à une idéologie qui se contente de prolonger et de radicaliser un humanisme largement indiscuté. L'augmentation des capacités humaines n'est un idéal que pour qui a adopté une perspective qui est celle de l'homme diminué. L'adoption d'une telle perspective n'est pas l'effet d'un choix volontaire et réfléchi: elle est l'effet de l'orientation prise par la modernité dont elle signe l'échec. Loin de rendre le monde plus accueillant, la mutation des techniques en technologies a entraîné une série de pertes. Les indéniables bénéfices en termes de bien-être matériel et d'expansion des libertés individuelles ne suffisent plus à les compenser, ni même à les masquer : diminution des facultés politiques par dissolution de la politique dans la technologie (p.42), précarité dans la sphère du travail, dévastation de l'environnement, « frustration imposée aux instincts communautaires » (p.54) dans un contexte où l'individu est censé être souverain, détresse généralisée. Dans ces conditions, les prétendues promesses transhumanistes ne sont pas autre chose qu'une «radicalisation de l'aliénation» (p.77) exprimant simplement l'exigence d'adaptation à un «environnement technologique si hégémonique qu'il ne respecte même plus l'intégrité corporelle» (p. 77).

Le dernier chapitre opère un basculement. Il s'agissait, dans le précédent, de décrire la condition diminuée d'un individu sans allégeances, devenu étranger à lui-même et ainsi vulnérable à la propagande transhumaniste. Il s'agit maintenant de remonter à la racine de ce mal, jusqu'à ses «déterminations plus profondes» (p.90). C'est la 
transformation du rapport au monde opérée par la modernité qui va être explorée et mise en cause. Elle est à chercher dans la façon dont la science moderne assimile le connaissable au calculable, à ce qui peut être exprimé de façon mathématique. Or, ce qui peut être exprimé en termes mathématiques, c'est d'abord l'inanimé. Dans une telle perspective, le vivant devient une source d'embarras et non un modèle pour la compréhension du monde. En témoignent les définitions contorsionnées que les biologistes et les épistémologues contemporains donnent de la vie. En témoigne également la façon dont la finalité est écartée de l'être vivant, réduit à un faisceau de fonctions séparées les unes des autres et dont chacune est susceptible d'être augmentée pour son propre compte. En témoigne encore la prévalence de la conception selon laquelle la nature est une machine. Reprenant probablement à son compte les analyses du juriste et historien du droit Michel Villey, O.Rey reconduit cette conception au débat médiéval sur la nature des universaux. Les nominalistes mettent l'accent sur la toute-puissance de Dieu plutôt que sur sa sagesse : dans une telle perspective, la création fait venir à l'existence une diversité de créatures singulières sans référence à une nature commune. En témoigne enfin la façon dont, dans une perspective darwinienne, est affirmée mais aussi neutralisée l'autonomie des organismes, engagés dans une lutte pour la survie et pour la propagation d'une descendance sans qu'une finalité intelligente joue le moindre rôle dans le processus. L'idée centrale est que la modernité purge le monde de ses fins et le réduit à n'être qu'un "réservoir de moyens au service des finalités humaines » (p. 159). O. Rey prône alors, à la suite de Robert Spaemann (philosophe et éthicien allemand, mort en 2018 qui a été membre de l'Académie pontificale pour la vie), une connaissance "sympathique » de la nature, une «connaissance qui ne serait pas au service d'un "faire" mais aiderait les hommes à comprendre leur situation dans le Tout du monde» (p. 149).

Un épilogue permet à l'auteur de s'interroger sur les rapports entre humanisme et transhumanisme. Il y affirme, face aux menaces qui viennent, la nécessité de recourir à des facultés et à des vertus très humaines.

Il faut être reconnaissant à $\mathrm{O}$. Rey de ne pas confondre, comme c'est encore trop souvent le cas, le posthumain des posthumanistes, qui prétendent avoir dépassé l'humanisme (quoi qu'ils entendent par là...) et le posthumain des transhumanistes, qui se donnent pour objectif de transcender l'humanité (et l'on ne comprend que trop bien, à la lecture de cet ouvrage, ce qu'ils peuvent entendre par là). O. Rey écrit superbement et directement, mais le lecteur est loin d'être toujours convaincu par son argumentation. Voici pourquoi : même s'il se défend d'avoir voulu écrire un pamphlet (p.11), l'auteur s'inscrit dans la tradition très française de l'essayiste imprécateur, de Léon Bloy jusqu'à Éric Zemmour. Cela le conduit, par exemple, à enrôler de force, au service de sa thèse le peintre Charles Sheeler dont l'admirable tableau American landscape méritait certainement mieux qu'un commentaire au premier degré, digne du pire Heidegger (p. 22-23). Si l'ouvrage n'est pas officiellement un pamphlet, il a bien souvent l'outrance du pamphlet. Par ailleurs, O. Rey se réclame de la sagesse de la répugnance de Leon Kass, un médecin et éthicien américain contemporain, considéré comme un représentant des «bioconservateurs». Mais, selon L. Kass lui-même, la sagesse de la répugnance ne constitue pas un argument. Elle rend moralement suspectes les tentatives d'argumenter contre certaines pratiques que le commun des mortels trouve spontanément et à juste titre répugnantes (inceste, cannibalisme, zoophilie, nécrophilie, clonage reproductif, etc.). Ces pratiques sont à ce point révoltantes qu'il est inutile et vain de présenter des arguments visant à les réfuter. Le problème, comme l'auteur le reconnait lui-même, est que ce qui paraît monstrueux à certains, comme la perspective de se voir envahi par la technologie, est au contraire ardemment désiré par d'autres (p. 78-79). Il se retrouve donc dans la situation d'argumenter contre les transhumanistes. Mais une sagesse de la répugnance qui cherche à argumenter se contredit ellemême. À la limite, les activistes grenoblois de Pièces et main d'œuvre (auxquels l'auteur semble vouer une sympathie certaine) sont plus cohérents : ils ne cherchent pas à argumenter contre les transhumanistes, mais simplement à tourner en dérision leur esprit de sérieux et à dénoncer leur goût du pouvoir (et de l'argent).

Écartelé qu'il est entre le style pamphlétaire et le style argumentatif, l'ouvrage de O. Rey n'en constitue pas moins un remarquable essai qui mérite d'être lu pour sa lucidité et son énergie.

Jean-Yves Goffi (Université Grenoble Alpes, IPhiG [ex-PPL], Grenoble, France) jean-yves.goffi@wanadoo.fr 